\title{
A new mixed-mode fracture criterion for large-scale lattice models
}

\author{
T. Sachau ${ }^{1}$ and D. Koehn ${ }^{2}$ \\ ${ }^{1}$ Institut für Geowissenschaften, Johannes Gutenberg-Universität Mainz, Mainz, Germany \\ ${ }^{2}$ School of Geographical and Earth Sciences, University of Glasgow, Glasgow, UK \\ Correspondence to: T. Sachau (till.sachau@gmail.com)
}

Received: 17 July 2013 - Published in Geosci. Model Dev. Discuss.: 7 August 2013

Revised: 30 December 2013 - Accepted: 7 January 2014 - Published: 29 January 2014

\begin{abstract}
Reasonable fracture criteria are crucial for the modeling of dynamic failure in computational lattice models. Successful criteria exist for experiments on the micro- and on the mesoscale, which are based on the stress that a bond experiences. In this paper, we test the applicability of these failure criteria to large-scale models, where gravity plays an important role in addition to the externally applied deformation. Brittle structures, resulting from these criteria, do not resemble the outcome predicted by fracture mechanics and by geological observations. For this reason we derive an elliptical fracture criterion, which is based on the strain energy stored in a bond. Simulations using the new criterion result in realistic structures. It is another great advantage of this fracture model that it can be combined with classic geological material parameters: the tensile strength $\sigma_{0}$ and the shear cohesion $\tau_{0}$. The proposed fracture criterion is much more robust with regard to numerical strain increments than fracture criteria based on stress (e.g., Drucker-Prager). While we tested the fracture model only for large-scale structures, there is strong reason to believe that the model is equally applicable to lattice simulations on the micro- and on the mesoscale.
\end{abstract}

\section{Introduction}

Fracturing caused by mechanical loading is the main reason for failure of brittle geological materials. The study of fracture formation and fracture propagation is therefore of enormous interest in order to understand the resulting structures. Lattice models allow a straightforward implementation of the material, including the material heterogeneity. Traditionally, these models have been applied to mechanical problems on the micro- or mesoscale.
Lattice models consist of a mesh of elastic bonds, which can be either regular or randomly irregular (e.g., Sachau and Koehn, 2012). Dynamic fracture processes can be reproduced by the sequential removal of these bonds from the lattice structure (Lilliu and van Mier, 2003). In the most basic setup only the normal force, acting on a bond, is taken into account (Ostoja-Starzewski, 2002). More sophisticated models consider also shear forces (Ostoja-Starzewski, 2002) and the rolling and torsional torques (Wang and AlonsoMarroquin, 2009). The choice of a specific model depends mainly on the experimental setup and on the computational resources.

It is crucial that these models apply a realistic failure criterion. In case of small-scale models, the fracture criterion is usually based on the tensile strength of the material (e.g., Flekkoy and Malthe-Sorenssen, 2002; Abe et al., 2006; Lilliu and van Mier, 2003; Schlangen and Garboczi, 1996), which assumes that local tensile failure is sufficient to model more complicated structures on the scale of the mesh size (Lilliu and van Mier, 2003).

Shear or mixed mode failure cannot be ignored in case of large-scale models (e.g., on the scale of the lithosphere or even on the scale of a geological outcrop (Schlangen and Garboczi, 1997)). Some existing small-scale models add a shear failure criterion to the tensile criterion in such a way that a bond is broken if either the shear strength or the tensile strength is exceeded (Zhao et al., 2012, 2010). Another potentially useful fracture criterion, which will be discussed in this paper, is the more sophisticated Drucker-Prager criterion.

However, in our large-scale numerical experiments none of these criteria produce realistic structures if applied to brittle large-scale setups, e.g., on the crustal scale (see Sect. 2 below). 
a)



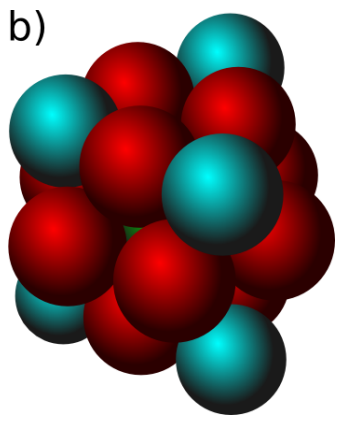

Fig. 1. Setup of the model. (a) The general structure of the model, which uses a regular hexagonal close-packed (HCP) geometry. Here, nodes are visualized as particles. (b) The next-nearest neighbor geometry of unit cells. A central node/particle is connected to its nearest neighbors (red) and its next-nearest neighbors (turquoise). As a result of the unit cell geometry, mesh bias is largely inhibited.

The failure criteria described above will be termed stressbased criteria in the following, in difference to criteria based on the strain energy.

The discussion in this paper concentrates on uniaxial extension experiments. The reason behind this decision is the very limited predictability of fault structures that form under compression. Even controlled analogue sandbox models have major difficulties in replicating experimental results (for instance Buiter et al., 2006), which suggests that the significance of a comparison between numerical and analogue results is limited.

\section{Background and test of stress-based fracture criteria}

We developed an isotropic lattice-particle model with regular hexagonal close-packed (HCP) geometry (Fig. 1a). Every node in the lattice structure is connected to its next neighbor node and to the second next neighbor node (Fig. 1b). This particular geometry is known to inhibit mesh effects almost completely (Sachau and Koehn, 2012), thus avoiding one of the largest problems in the application of lattice models to fracture problems.

The connecting elements between nodes are onedimensional linear elastic bonds. These bonds are similar to elastic normal springs, but exert shear forces in addition to normal forces. For each bond, the shear force and the normal force can be calculated from the relative displacement of nodes with regard to a relative equilibrium position (see Fig. 2). Nodal displacement is primarily caused by externally applied deformation or by dynamic internal processes such as fracturing. Macroscopic elastic parameters of the lattice are controlled by constants, which relate the normal force and the shear force to the nodal displacement. Details of the underlying mathematics are given in Sachau and Koehn (2012).

If a bond exceeds a given fracture criterion, it is removed from the system and a fracture is formed. The particular

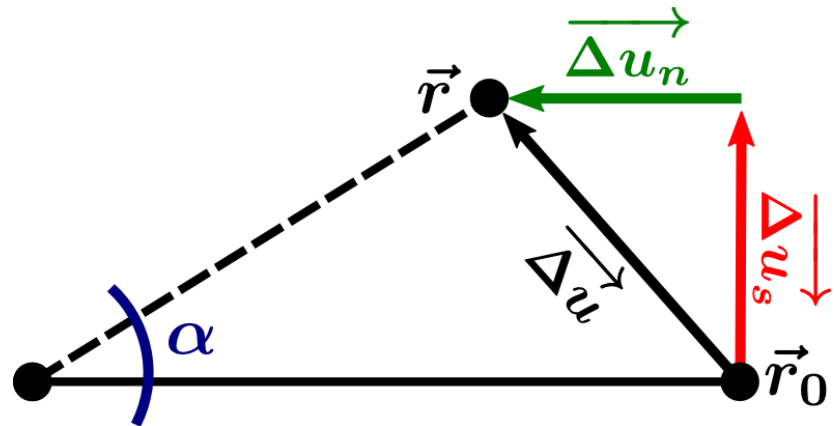

Fig. 2. Relative nodal displacement. $\overrightarrow{r_{0}}$ is the equilibrium position of a node with respect to a neighbor node. From the relative displacement $\overrightarrow{\Delta u}$, the normal displacement $\overrightarrow{\Delta u_{\mathrm{n}}}$ and the shear angle $\alpha /$ shear displacement $\overrightarrow{\Delta u_{\mathrm{s}}}$ can be calculated. Finally, the shear force and the normal force can be derived from $\overrightarrow{\Delta u_{\mathrm{n}}}$ and from $\overrightarrow{\Delta u_{\mathrm{s}}}$.

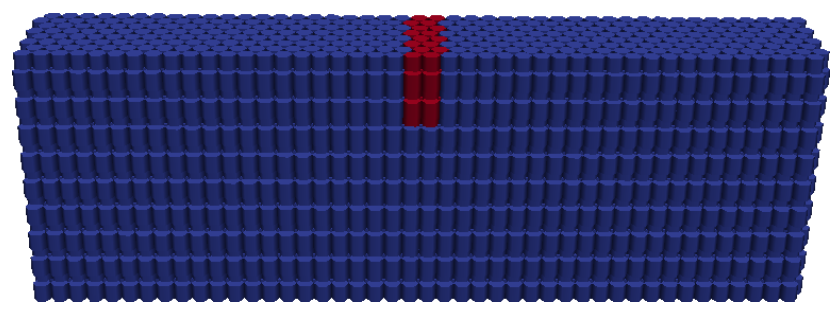

Fig. 3. Typical model setup used for most experiments in this paper. Particles next to fractures are displayed in red. A vertical fault with a height of ca. $5 \mathrm{~km}$ is inserted close to the surface in order to trigger localization. Model scale is $30 \mathrm{~km} \times 60 \mathrm{~km} \times 10 \mathrm{~km}$, density $\rho=2600 \mathrm{~kg} \mathrm{~m}^{-3}$, the Young modulus $E=100 \mathrm{GPa}$, and the Poisson ratio $v=0.2$. Tensile breaking strength $\sigma_{0}=50 \mathrm{MPa}$; angle of internal friction $\psi=34^{\circ}$.

geometry of the lattice generates elastic isotropy of the lattice, which means that the growth of fractures is entirely controlled by the conditions of the experiment (i.e., by the fracture criterion, the material parameters and the details of the external deformation, and not by inherent anisotropy).

If not explicitly stated otherwise, the experiments shown in this paper have usually the following setup: the size of the model is on the crustal scale with $30 \mathrm{~km} \times 60 \mathrm{~km} \times 10 \mathrm{~km}$. The model is fully elastic and is subjected to step-wise uniaxial horizontal extension as well as to its own gravitational load. A single strain increment is $60 \mathrm{~m}$, and the total strain, at which the fracture network is evaluated, amounts to $2.4 \mathrm{~km}$, corresponding to a strain of $\epsilon=0.04$. The density $\rho$ of the material is $2600 \mathrm{~kg} \mathrm{~m}^{-3}$, and the Young modulus $E=100 \mathrm{GPa}$, the Poisson ratio $v=0.2$. The tensile breaking strength $\sigma_{0}=50 \mathrm{MPa}$, and the angle of internal friction $\psi=34^{\circ}$. In order to improve the localization of fractures, a small vertical fault is inserted prior to the extension (Fig. 3).

In the simulation shown in Fig. $4 \mathrm{a}$, bonds fail once a critical tensile stress is exceeded. As a result of the uniaxial 

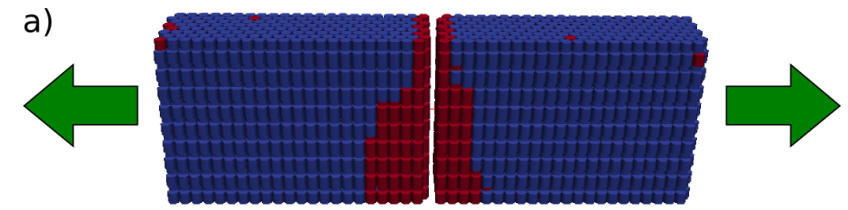

b)
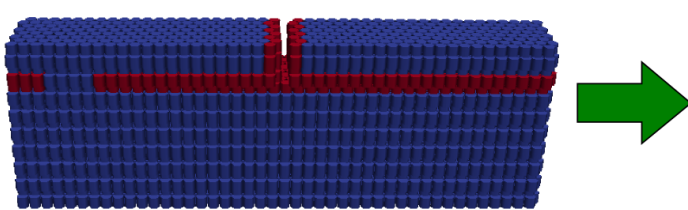

Fig. 4. Uniaxial horizontal extension of a brittle crust with two different stress-based fracture criteria. Both simulations consider the gravitational load. For a detailed description of the setup, compare Fig. 3 and Sect. 2. The figure shows the simulation at a tensile strain of 0.04 , equivalent to $2.4 \mathrm{~km}$ extension. Particles next to broken bonds are red. Bonds break in (a) if the tensile stress $\sigma_{\mathrm{n}}$ exceeds a given threshold $\sigma_{0}$ and in (b) if either $\sigma_{\mathrm{n}}$ or the shear stress $\sigma_{\mathrm{s}}$ exceeds $\sigma_{0}$ or $\tau_{0}$. None of the results resembles the expected graben structure.

extension, the entire crust is severed by a single vertical fault. In Fig. 4b failure occurs if either a critical shear stress or a critical tensile stress is exceeded. As a consequence a horizontal shear fault is created, which separates the entire uppermost crust from the lower crust.

None of the fault structures resulting from these classical stress-based criteria resembles a graben structure, which would be expected by fracture mechanical considerations and geological observations (e.g., Sun and Jin, 2011; Gudmundsson, 2012) for the given type of external deformation.

We must therefore conclude that stress-based fracture criteria are not always adequate for the modeling of large-scale failure in brittle materials. The problem is generally of lesser significance if such criteria are applied to model the deformation of a layered crust or to heterogeneous bodies. See discussion in Sect. 4 below.

\section{New fracture criterion}

In order to improve the failure behavior, we propose a new fracture criterion based on the strain energy stored in each bond instead of stress. In the failure models described above, breaking occurs if either the shear stress $\tau$ or the tensile normal stress $\sigma_{\mathrm{t}}$ reaches a critical value (Fig. 5a). From the results above it becomes clear that a better link between these fracture criteria is needed in order to generate more realistic structures.

For this purpose we propose an elliptical energy model for mixed mode failure. The total strain energy $U_{\text {tot }}$ in a deformed body can be calculated by
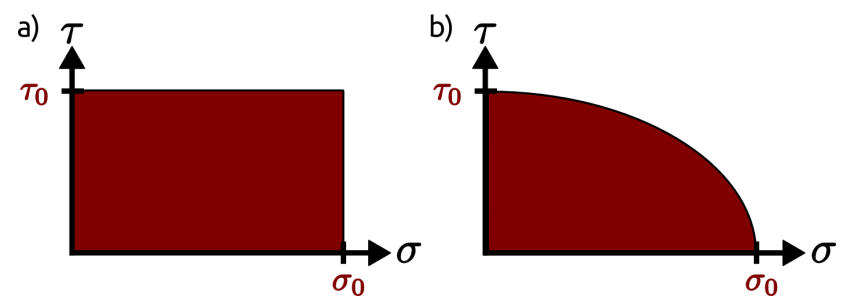

Fig. 5. The stress-based criterion (a) compared to the elliptical criterion based on strain energy (b). Axes are for shear stress $\tau$ and tensile stress $\sigma$; the respective yield values are $\tau_{0}$ and $\sigma_{0}$. Fracturing occurs if the state of stress of a bond plots outside the marked area. If $\tau_{0} \neq \sigma_{0}$, then (b) represents Eq. (3). If $\tau_{0}=\sigma_{0}$, (b) is a circle representing Eq. (2).

$U_{\mathrm{tot}}=U_{\mathrm{t}}+U_{\mathrm{s}}$,

where $U_{\mathrm{t}}$ and $U_{\mathrm{s}}$ are the strain energies related to tension and shear.

If we assume a single critical value for the strain energy $E_{\mathrm{c}}$, then failure occurs if $E_{\mathrm{c}}=U_{\text {tot }}$. After substitution into Eq. (1) and rearrangement, the failure criterion becomes

$\frac{U_{\mathrm{s}}+U_{\mathrm{t}}}{E_{\mathrm{c}}}=1$.

We can include separate parameters for the critical energies related to shear $\left(E_{\mathrm{c}, \tau}\right)$ and to tension $\left(E_{\mathrm{c}, \sigma}\right)$ instead of using the general value $E_{\mathrm{c}}$ by introducing the following criterion:

$\frac{U_{\mathrm{s}}}{E_{\mathrm{c}}}+\frac{U_{\mathrm{t}}}{E_{\mathrm{c}}}=\left(\frac{\sigma_{n}}{\sigma_{0}}\right)^{2}+\left(\frac{\tau}{\tau_{0}}\right)^{2}=1$,

which describes an ellipse in $\sigma_{\mathrm{n}}-\tau$ space (cf., e.g., Sun and Jin, 2011, for this operation).

The following applies to both Eqs. (2) and (3): if there is no contribution of either the shear or the normal stress (meaning $\sigma_{\mathrm{n}}=0$ or $\tau=0$ ), then the equation reduces to $\sigma_{\mathrm{n}} / \sigma_{0}=1$ or $\tau / \tau_{0}=1$, respectively. This is equivalent to tensile failure or shear failure, just as in simple stress-based models. Therefore, the critical values for the strain energy related to either shear $\left(E_{\mathrm{c}, \tau}\right)$ or tension $\left(E_{\mathrm{c}, \sigma}\right)$ can be calculated from the tensile strength $\sigma_{0}$ and the shear cohesion $\tau_{o}$ of the material:

$E_{\mathrm{c}, \tau}=\frac{\tau_{0}}{2 G}, \quad E_{\mathrm{c}, \sigma}=\frac{\sigma_{0}}{2 E}$.

$G$ and $E$ are the shear modulus and Young's modulus.

If this criterion is applied to dynamic fracture simulations with sequential removal of bonds, Eq. (3) can be interpreted as a probability. The bond with the highest result $>1$ is removed from the network, and the stress field is recalculated. The process is successively repeated until no bond with a probability $>1$ remains. 


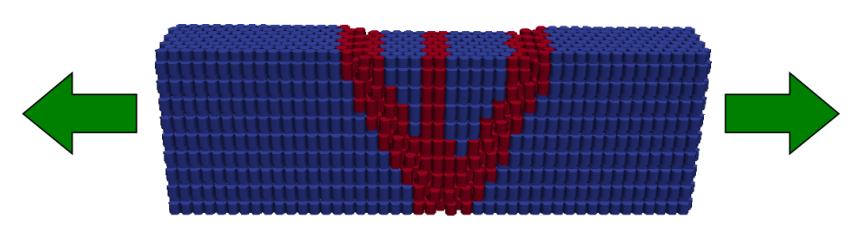

Fig. 6. Application of the new mixed mode fracture criterion in a setup similar to the crustal-scale simulations shown in Fig. 4. The tensile strain is $0.04(2.4 \mathrm{~km})$. Particles next to fractures are red. The fault network forms a graben structure, with the surface subsiding along the fault planes. This is the structure that fracture mechanics and geological observations predict.

\section{Discussion}

Figure 6 displays the results of a simulation where the new criterion is applied. The setup is identical to the one shown in Sect. 2. The resulting fault network resembles a graben structure and is in line with considerations from fracture mechanics and structural geology.

The accuracy of the criterion was tested in a number of simulations with varying values for the angle of internal friction $(\psi)$. Using $\psi$, the angle $\alpha$ between a fault plane and the orientation of the maximum principal stress $\sigma_{1}$ can be calculated from Coulomb theory (e.g., Gudmundsson, 2012) by

$\alpha=45^{\circ}-\frac{\psi}{2}$.

The experimental setup is identical to the previous experiment. The orientation of $\sigma_{1}$ is given by the direction of the gravity force, the orientation of $\sigma_{3}$ by the direction of the uniaxial extension. The fault planes for $\psi=30^{\circ}$ and $\psi=45^{\circ}$ are displayed in Fig. 7a and b. The expected inclination $\alpha=30^{\circ}$ for $\psi=30^{\circ}$, compared to $29.3^{\circ}$ in the experiment (Fig. 7a). For $\psi=45^{\circ}$ the calculated angle $\alpha$ equals $22.5^{\circ}$, compared to $21.8^{\circ}$ in the simulation (Fig. $7 \mathrm{~b}$ ). Note that it is difficult to assess the exact inclination of a plane in a lattice-particle model, due to the general roughness of surfaces.

Figure $7 \mathrm{c}$ to $\mathrm{f}$ compare these results to fault planes generated by alternative fracture criteria.

The Drucker-Prager criterion has been tested with $\psi=$ $30^{\circ}$ (Fig. 7c and d). The accuracy is low in comparison to the strain energy criterion: $\alpha=22.6^{\circ}$, compared to an expected value $\alpha=30^{\circ}$. Convergence to a stable solution requires relatively small strain increments. The strain energy criterion generates stable results at $\Delta s \approx 10^{-3}$, compared to $\Delta s \approx 10^{-5}$ if the Drucker-Prager criterion is applied.

The popular tensile stress criterion (Fig. 7e) results in a vertical fault plane, the shear stress criterion (Fig. 7f) in a horizontal fault plane. These criteria must be considered unsuitable for the simulation of realistic geological structures.

Finally, we tested the geologically important transition from vertical fault planes to inclined faults with depth under


Fig. 7. Inclination of fault planes resulting from uniaxial extension. Results using the proposed fracture criterion are compared with results using the Drucker-Prager criterion, the tensile stress and the shear stress criterion. Colors indicate the layering of the model. $\sigma_{1}$ is vertical, given by gravity. $\alpha$ is the angle between the fault plane and $\sigma_{1} . \Delta s$ is the strain increment per time step.

Structures formed with the proposed fracture criterion converge at relatively large strain increments $\left(\Delta s \approx 10^{-3}\right)$, compared to the Drucker-Prager criterion $\left(\Delta s \approx 10^{-5}\right)$. The fault plane inclination is far closer to expectation values when using the new criterion compared to other fracture criteria.

(a, b) Proposed fracture criterion. (a) $\phi=30^{\circ}, \alpha=29.3^{\circ}$ compared to $\alpha=30^{\circ}$ if calculated from Eq. (5). (b). $\phi=45^{\circ}, \alpha=22.5^{\circ}$ versus $21.8^{\circ}$ measured.

(c, d) Drucker-Prager criterion. Strain increment dependency: inclination of fault planes converges at $\Delta s \approx 10^{-5}$, vs. $\Delta s \approx 10^{-3}$ using the proposed criterion. $\phi=30^{\circ}$ in both (c) and (b). Expected $\alpha$-value $=30^{\circ}$, measured value $=22.6^{\circ}$.

(e, f) Shear stress (e) and tensile stress (f) criteria. Fault planes are perpendicular or parallel to the extension.

tensile conditions. The corresponding numerical experiment is displayed in Fig. 8. Close to the surface, shear stress is comparatively low, resulting in vertical tensile fractures. Shear stress increases with the gravitational load, which leads to shear failure and thus inclined fracture planes at greater depth. We used the same setup as in Fig. 3, but with a 


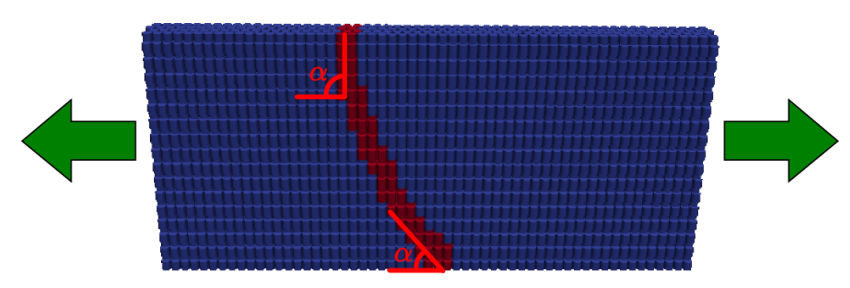

Fig. 8. Transition from tensile vertical faults to inclined shear faults with depth. The setup applies uniaxial tension to a similar setup as in the reference setup in Fig. 3, but with a reduced height of just $15 \mathrm{~km}$. The angle of the fault plane to the horizontal $(\alpha)$ is $90^{\circ}$ at the surface and about $45^{\circ}$ at the bottom of the model. Extension of the model is 0.02 , equivalent to $0.6 \mathrm{~km}$.

reduced height of only $15 \mathrm{~km}$. The height reduction is necessary because tensile faults in the crust occur only to a depth of a few kilometers.

The result of the simulation is in agreement with the expectations. The angle between fault plane and a horizontal plane decreases from $90^{\circ}$ at the model surface to about $45^{\circ}$ in $15 \mathrm{~km}$ depth.

The effect of the strain-based criterion, compared to stressbased criteria, is less significant in the case of layered materials or materials with strong heterogeneities. Examples are models of crustal extension, which include the brittle ductile transition or materials with strong heterogeneities. We recalculated crustal-scale experiments with a brittle elastic crust from previous publications (Sachau and Koehn, 2010, 2012, and Sachau et al., 2013), with the same results as previously published.

\section{Conclusions}

In this article we derived an elliptical fracture model for lattice models, based on the strain energy of bonds connecting nodes. The model is capable of incorporating classical geological yield limits for shear stress and tensile stress. The fracture model has been tested in a variety of tensile crustalscale simulations, using a numerical 3-D lattice model. In these tests we compare the structures that develop in a model with a stress-based criteria with those that develop when the fracture criterion is based on strain energy.

Crustal-scale structures, which have been modeled with the new strain-based criterion, have far more resemblance with the geological reality and with the predictions of fracture mechanics than structures resulting from stress-based criteria like the tensile stress criterion and the DruckerPrager criterion. The inclination of fault planes is reasonably accurate if compared to values predicted by the Coulomb criterion. The inclination of fault planes increases with depth, due to increase in shear stress.

The required size of strain increments to produce stable results is much larger than for the Drucker-Prager criterion. This has positive effects on the computation time.
The new criterion is particularly interesting for exclusively brittle model setups, which do not include other effects, like viscoelastic behavior near the brittle ductile transition zone. Also mesh effects may influence the geometry of fractures, thereby diminishing the role of the fracture criterion. We do, nevertheless, strongly recommend the application of the new criterion in any lattice simulation involving brittle fracture.

Acknowledgements. We acknowledge funding by the Deutsche Forschungsgemeinschaft (DFG), grant KO 2463/4-2.

Edited by: L. Gross

\section{References}

Abe, S., Latham, S., and Mora, P.: Dynamic Rupture in a 3-D Particle-based Simulation of a Rough Planar Fault, Pure Appl. Geophys., 163, 1881-1892, 2006.

Buiter, S., Babeyko, A., Ellis, S., Gerya, T., Kaus, B., Kellner, A., Schreurs, G., and Yamada, Y.: The numerical sandbox: comparison of model results for a shortening and an extension experiment, Geological Society, London, Special Publications, 253, 29-64, 2006.

Flekkoy, E. G. and Malthe-Sorenssen, A.: Modeling hydrofracture, J. Geophys. Res., 107, 1-11, 2002.

Gudmundsson, A.: Rock Fractures in Geological Processes, Cambridge University Press, Cambridge, 2012.

Lilliu, G. and van Mier, J. G. M.: 3D lattice type fracture model for concrete, Eng. Fract. Mech., 70, 927-941, 2003.

Ostoja-Starzewski, M.: Lattice models in micromechanics, Appl. Mech. Rev., 55, 35-60, 2002.

Sachau, T. and Koehn, D.: Faulting of the lithosphere during extension and related rift-flank uplift: a numerical study, Int. J. Earth Sci., 99, 1619-1632, 2010.

Sachau, T. and Koehn, D.: "Melange": A viscoelastic lattice-particle model applicable to the lithosphere, Geochem. Geophy. Geosys., 13, Q12009, doi:10.1029/2012GC004452, 2012.

Sachau, T., Koehn, D., and Passchier, C.: Mountain-Building Under Extension, Am. J. Sci., 313, 326-344, 2013.

Schlangen, E. and Garboczi, E. J.: New method for simulating fracture using an elastically uniform random geometry lattice, Int. J. Eng. Sci., 34, 1131-1144, 1996.

Schlangen, E. and Garboczi, E.: Fracture simulations of concrete using lattice models: Computational aspects, Eng. Fract. Mech., 57, 319-332, 1997.

Sun, C. and Jin, Z.: Fracture Mechanics, Academic Press, Waltham, 2011.

Wang, Y. and Alonso-Marroquin, F.: A finite deformation method for discrete modeling: particle rotation and parameter calibration, Granular Matter, 11, 331-343, 2009.

Zhao, G.-F., Fang, J., and Zhao, J.: A 3D distinct lattice spring model for elasticity and dynamic failure, Int. J. Numer. Anal. Met., 35, 859-885, doi:10.1002/nag.930, 2010.

Zhao, G.-F., Khalili, N., Fang, J., and Zhao, J.: A coupled distinct lattice spring model for rock failure under dynamic loads, Comput. Geotech., 42, 1-20, 2012. 